BULL. AUSTRAL. MATH. SOC.

VOL. 19 (1978), 59-65.

\title{
Inverse semigroups and their natural order
}

\section{H. Mitsch}

The natural order of an inverse semigroup defined by $a \leq b \Longleftrightarrow a^{\prime} b=a^{\prime} a$ has turned out to be of great importance in describing the structure of it. In this paper an ordertheoretical point of view is adopted to characterise inverse semigroups. A complete description is given according to the type of partial order an arbitrary inverse semigroup $S$ can possibly admit: a least element of $(S, \leq)$ is shown to be the zero of $(S, \cdot)$; the existence of a greatest element is equivalent to the fact, that $(S, \cdot)$ is a semilattice; $(S, \leq)$ is directed downwards, if and only if $S$ admits only the trivial group-homomorphic image; $(S, \leq)$ is totally ordered, if and only if for all $a, b \in S$, either $a b=b a=a$ or $a b=b a=b ; a$ finite inverse semigroup is a lattice, if and only if it admits a greatest element. Finally formulas concerning the inverse of a supremum or an infimum, if it exists, are derived, and rightdistributivity and left-distributivity of multiplication with respect to union and intersection are shown to be equivalent.

\section{Introduction}

Let $(S, \cdot)$ be an inverse semigroup, $(E, \cdot)$ the lower semilattice of all idempotent elements of $S$. Then a partial order " $\leq$ " - the socalled "natural order" - can be defined on $S$ by

$$
a \leq b \Longleftrightarrow a b^{\prime}=a a^{\prime}
$$

Received 15 June 1978. 
( $a$ ' denotes the unique inverse of $a$ in $S$ ); the order "s" on $S$ restricted to $E$ coincides with the partial order of the lower semilattice $E: e \leq f \Longleftrightarrow e=e f=f e(e, f \in E)$. Several equivalent formulations of this definition are known (see $[2,3],[6])$ :

$$
\begin{aligned}
& a \leq b \Longleftrightarrow a^{\prime} b=a^{\prime} a, \\
& a \leq b \Longleftrightarrow a=e b, \quad e \in E .
\end{aligned}
$$

With respect to this order, $(S, \cdot)$ forms a partially ordered semigroup, that is to say, $a \leq b \Rightarrow a c \leq b c$ and $c a \leq c b$ for all $c \in S$; hence also $a \leq b \Longleftrightarrow a^{\prime} \leq b^{\prime}$ (see [2,3], [5]). The natural order on $S$ is even a special "natural" ordering in the sense of partially ordered semigroups (see [5]):

$$
a \leq b \Leftrightarrow a=\left(a a^{\prime}\right) b=b\left(a^{\prime} a\right) \text {. }
$$

In fact, by (2), $a \leq b$ implies $a^{\prime} b=a^{\prime} a$, thus, multiplying by $a$ on the left, $\left(a a^{\prime}\right) b=a$; conversely $a=\left(a a^{\prime}\right) b=e b$ with $e \in E$ implies $a \leq b$, by (3). Furthermore

$$
a \leq b \Longleftrightarrow a^{\prime} \leq b^{\prime} \Longleftrightarrow a^{\prime}=\left(a^{\prime} a\right) b^{\prime} \Leftrightarrow a=b\left(a^{\prime} a\right)
$$

by taking inverses.

Order-theoretic considerations have turned out to be of great importance in the theory of inverse semigroups. In the following a description of special types of partial orderings which the natural order on an inverse semigroup can possibly assume will be given.

\section{Special natural orders}

In every inverse semigroup $S$ we have, with respect to its natural order,

$$
a \leq e, \quad e \in E, \quad a \in S \Rightarrow a \in E
$$

(by (3), $a=f e, f \in E$, thus $a \in E$ );

$$
e \leq a, e \in E, a \in S \Rightarrow e a=a e=e
$$

(by (4), $\left.e=\left(e e^{\prime}\right) a=e a, e=a\left(e^{\prime} e\right)=a e\right)$.

LEMMA 1. An element a of an inverse semigroup $S$ is the least element of $S$, if and only if $a$ is the zero of $S$.

Proof. If $c$ is the zero of $S$, then $c=c a$ for all $a \in S$ and 
$c \in E$; hence by (3), $c \leq a$ for all $a \in S$. Conversely if $c \leq a$ for all $a \in S$, then, by (4), $c=\left(c c^{\prime}\right) a=a\left(c^{\prime} c\right)$. If there is $e \in E$ (that is if $E \neq \emptyset$ ), then with $a=e$ we obtain $c=\left(c c^{\prime}\right) e \in E$ and $c=c a=a c$ for all $a \in S$; if $E=\emptyset$, then $S$ can not be an inverse semigroup, since $a \in S$ implies $a a^{\prime} \in E$. / /

LEMMA 2. An inverse semigroup $S$ possesses a greatest element, if and only if $S$ is an idempotent, commutative semigroup with identity (that is a semilattice).

Proof. If $S$ is a semilattice with identity $e$, then $a=a e$ for all $a \in S$; since $S=E$, we conclude from (3) that $a \leq e$ for all $a \in S$.

Conversely assume $a \leq i$ for all $a \in S$ (where $i$ is the greatest element of $S$ ); then by (I), $a i^{\prime}=a a^{\prime}$ for all $a \in S$. For $e \in E$ we have $e i^{\prime}=e$, thus $e\left(i^{\prime} i\right)=e i$; but $e, i^{\prime} i \in E$ implies $e i \in E$. Now if $a \in S$ is an arbitrary element, then $a \leq i$ implies, by (4), $a=\left(a a^{\prime}\right) i$ with $a a^{\prime} \in E$; consequently $a \in E$ for all $a \in S$. This means that $S$ is an idempotent, and thus commutative, semigroup (since idempotents commute in an inverse semigroup). Furthermore $a^{\prime}=a$ for all $a \in S$ and $a \leq i$ implies $a i=i a=a$ for all $a \in S$, and $i$ is the identity of $S$. //

A partial order " $\leq "$ on a set $S$ is called directed dowrwards, if for all $x, y \in S$ there exists a $z \in S$ with $z \leq x$ and $z \leq y$; the dual concept is directed upwards.

REMARKS. (1) If an inverse semigroup $S$ is directed upwards with respect to its natural order, then it is also directed downwards. In fact: if for all $x, y \in S$ there is $z \in S$ such that $x \leq z$ and $y \leq z$, then, by (3), $x=e z$ and $y=f z$, with $e, f \in E$; hence $f x=\dot{f} e z=e f z=e y=u \in S$, which means, again by (3), $u \leq x$ and $u \leq y$, and $S$ is directed downwards.

(2) If $S$ is directed downwards, then $S$ is a reversible semigroup $([2,3])$. In fact, if for all $x, y \in S$ there is $z \in S$ with $z \leq x$ and $z \leq y$, then, by (4), $z=e x=f y$ with $e, f \in E \subseteq S$, and $z=x g=y h$ with $g, h \in S$, which means $(S x) \cap(S y) \neq \emptyset$ and $(x S) \cap(y S) \neq \emptyset$.

LEMMA 3. An inverse semigroup $S$ is directed downwards with respect 
to its natural order, if and only if the right-reversible equivalence $P_{E}$ of Dubreil, defined by $a \equiv b\left(P_{E}\right) \Leftrightarrow e a=f b$ for some $e, f \in E$, is the universal relation.

Proof. $S$ is directed downwards if and only if for all $x, y \in S$, there exists a $z \in S$ with $z \leq x$ and $z \leq y$, or equivalently $z=e x=f y$ with $e, f \in E$, or equivalently again, for all $x, y \in S$, there exist $e, f \in E$ with $e x=f y$, or finally equivalently $P_{E}$ is the universal relation on $S$ (see $[2,3],[4])$. //

THEOREM 4. An inverse semigroup $S$ is directed downards with respect to its natural order, if and only if the only group-homomorphic image of $S$ is the trivial one.

Proof. By [7] the least group-congruence on an arbitrary inverse semigroup $S$ (that is to say, a congruence $\rho$ such that $S / \rho$ is a group) is defined by $a \equiv b(\rho)$ if and only if $e a=e b$ for some $e \in E$. If $S$ is directed downwards, then this is equivalent to the existence of $z \in S$, given $x, y \in S$, such that $z=\left(z z^{\prime}\right) x=\left(z z^{\prime}\right) y$ (by (4)), which means that $e x=e y$ for $e=z z^{\prime} \in E$. But this is equivalent to the fact that the least group-congruence on $S$ is the universal relation, which means that the maximal group-homomorphic image of $S$ is the trivial group; in other words there is no other group homomorphic to $S$ except the trivial one. $/ 1$

REMARK. As a consequence we note that inverse semigroups the natural order of which is an upper-semilattice, a lower-semilattice; or a latticeorder, necessarily have only the trivial group-homomorphic image.

One order-theoretical extreme is the case where the natural order of $S$ is a total order; we prove:

THEOREM 5. An inverse semigroup $S$ is totally ordered with respect to its natural ordering, if and only if $a b=b a=a$ or $a b=b a=b$ for $a \imath 2 \quad a, b \in S$.

Proof. If $a b=b a=a$ or $a b=b a=b$ for $a l l a, b \in S$, then for $b=a$ we get $a^{2}=a, a^{\prime}=a$ for all $a \in S$ and $E=S$. Thus for arbitrary $a, b \in S$ we have: if $a=a b$ then $a \leq b$, and if $b=a b=b a$, then $b \leq a$ by (3), and (S, $\leq)$ is totally ordered. 
Conversely if the natural order of $S$ is total, then we have $a \leq a^{\prime}$ or $a^{\prime} \leq a$ for all $a \in S$. By taking inverses we obtain $a^{\prime} \leq a$ or $a \leq a^{\prime}$; thus $a^{\prime}=a$ for all $a \in S$ and $a=a a^{\prime} a=a^{3}$ for all $a \in S$. Since $a^{2} \leq a$ or $a \leq a^{2}$ for all $a \in S$, we get, multiplying by $a, a=a^{3} \leq a^{2}$ or $a^{2} \leq a^{3}=a$; hence $a^{2}=a$ for all $a \in S$, and $E=S$. But idempotents of an inverse semigroup commute, so that $a b=b a$ for all $a, b \in S$. Furthermore $a \leq b$ or $b \leq a$ for all $a, b \in S$ implies, by (4), $a=\left(a a^{\prime}\right) b=a^{2} b=a b$ or $b=b a$ for all $a, b \in S$, and we conclude that $a b=b a=a$ or $a b=b a=b$ for all $a, b \in S$. //

REMARK. If an inverse semigroup $S$ possesses an identity $e$, then $e$ is maximal with respect to the natural order of $S$. In fact suppose $e<a$ for an $a \in S$; then, by (1), ea' $=e e^{\prime}=e^{2}=e$; thus $a^{\prime}=e$ and $a=\left(a^{\prime}\right)^{\prime}=e$, a contradiction. Furthermore if $e$ is the least element of $S$, then $S=\{e\}$.

Concerning semilattice-orders, Lemma 2 solves the problem on the assumption that there is a greatest element. Equivalently we state

LEMMA 6. Let $S$ be an inverse semigroup with identity $e$. Then $S$ is a lower semilattice with respect to its natural order, if $e$ is the greatest element of $S$.

Finally we give an answer to the question: which are the finite inverse semigroups whose natural order is a lattice-order?

THEOREM 7. Let $S$ be a finite inverse semigroup. Then $S$ is a lattice with respect to its natural order, if and only if $S$ possesses a greatest element.

Proof. If $S$ has a greatest element, then, by Lemma $2,(S, \leq)$ is a lower semilattice with respect to the operation " $\wedge$ " defined by $a \wedge b=a b$ for all $a, b \in S$ (see Theorem 1.12 of [2]). Since $(S, \leq$ ) is bounded from above, and since every non-void subset $T$ of $S$ has an infimum, inf $T=a_{1} \ldots a_{n}$ with $a_{i} \in T$, by a well-known result of lattice-theory every non-void subset of $S$ has also a supremum; in particular $\sup \{a, b\}=a \vee b=c_{1} \cdots c_{k}$ where $c_{i}$ runs through all elements of $S$, which are upper bounds for $\{a, b\}$. The converse is trivial, since a finite lattice has a greatest element. 
COROLLARY 8. Let $S$ be a finite inverse semigroup with identity $e$. Then $S$ is a lattice with respect to its natural order, if and only if $e$ is the greatest element of $S$.

Concerning possibly existing infima and suprema in inverse semigroups we show:

LEMMA 9. Let $S$ be an inverse semigroup. If $a, b$ are elements of $S$ for which $\sup \{a, b\}=a \vee b \quad(\inf \{a, b\}=a \wedge b)$ exists, then $a^{\prime} \vee b^{\prime}$ exists, too, and $(a \vee b)^{\prime}=a^{\prime} \vee b^{\prime}\left(a^{\prime} \wedge b^{\prime}\right.$ exists, too, and $\left.(a \wedge b)^{\prime}=a^{\prime} \wedge b^{\prime}\right)$.

Proof. If $a \vee b$ exists for $a, b \in S$, then $a, b \leq a \vee b$ and $a^{\prime}, b^{\prime} \leq(a \vee b)^{\prime}$; if $x \in S$ is any upper bound for $\left\{a^{\prime}, b^{\prime}\right\}$, then $a^{\prime}, b^{\prime} \leq x$ implies $a, b \leq x^{\prime}$; thus $a \vee b \leq x^{\prime}$ and $(a \vee b)^{\prime} \leq x$, that is $(a \vee b)^{\prime}$ is the least upper bound for $\left\{a^{\prime}, b^{\prime}\right\}$, and $(a \vee b)^{\prime}=a^{\prime} \vee b^{\prime}$. //

LEMMA 10. Let $S$ be an inverse semigroup which is a lattice with respect to its natural order. Then multiplication is left-distributive with respect to union (intersection), if and only if it is rightdistributive with respect to union (intersection).

Proof. Suppose $a(b \vee c)=a b \vee a c$ for all $a, b, c \in S$. Since $a, b \leq a \vee b$ implies $a c, b c \leq(a \vee b) c$, we have that $a c \vee b c \leq(a \vee b) c$ for all $a, b, c \in S$. Consequently in order to prove right-distributivity we have to show the converse inequality

$$
\begin{aligned}
{[(a \vee b) c](a c \vee b c)^{\prime} } & =[(a \vee b) c]\left[(a c)^{\prime} \vee(b c)^{\prime}\right] \text { by Lemma } 9 \\
& =[(a \vee b) c]\left(c^{\prime} a^{\prime} \vee c^{\prime} b^{\prime}\right) \\
& =[(a \vee b) c]\left[c^{\prime}\left(a^{\prime} \vee b^{\prime}\right)\right] \text { by assumption } \\
& =[(a \vee b) c][(a \vee b) c]^{\prime} .
\end{aligned}
$$

By (1) this means $(a \vee b) c \leq a c \vee b c$ for all $a, b, c \in S$. The proof of the converse statement is similar.

\section{References}

[1] Garrett Birkhoff, Lattice theory, Third edition (American Mathematical Society Colloquium Publications, 25. American Mathematical Society, Providence, Rhode Island, 1967). 
[2] A.H. Clifford and G.B. Preston, The algebraic theory of semigroups, Volume I (Mathematical Surveys, 7. American Mathematical Society, Providence, Rhode Island, 1961; reprinted 1964).

[3] A.H. Clifford and G.B. Preston, The algebraic theory of semigroups, Volume II (Mathematical Surveys, 7. American Mathematical Society, Providence, Rhode Island, 1967; reprinted 1971).

[4] Paul Dubreil, Contribution à Za théorie des semi-groupes (Mén. Acad. Sci. Inst. France (2) 63, no. 3, 52 pp (1941)).

[5] L. Fuchs, Partially ordered algebraic systems (Pure and Applied Mathematics, 28. Akadémiai Kiadó, Budapest; Pergamon Press, Oxford, London, New York, Paris, 1963).

[6] J.M. Howie, An introduction to semigroup theory (London Mathematical Society Monographs, 7. Academic Press (Harcourt Brace Jovanovich], London, New York, San Francisco, 1976).

[7] W.D. Munn, "A class of irreducible matrix representations of an arbitrary inverse semigroup", Proc. Glasgow Math. Assoc. 5 $(1961-62), 41-48$.

[8] В.В. Вагнер [V.V. Vagner], "Обобценные грутлы" [Generalized groups], Dokz. Akad. Nauk SSSR (N.S.) 84 (1952), 1119-1122.

Mathematisches Institut der Universität Wien, Wien, Austria. 